www.nature.com/pj

\title{
Rewritable holograms based on azobenzene-containing liquid-crystalline polymers
}

\begin{abstract}
Atsushi Shishido
The design and preparation of azobenzene-containing polymers showing a large photoinduced change in refractive index, and their applications to rewritable Raman-Nath and Bragg holograms are reviewed. Optically transparent films with a thickness of $>200 \mu \mathrm{m}$ have been prepared with random copolymers, which contain photoresponsive and amplifying components in the side chain. Various azobenzene chromophores are used as the photoresponsive component. Cyanobiphenyl and tolane moieties are incorporated as the amplifying component, which enhances the change in refractive index triggered by the azobenzene. When two writing beams are made to overlap in the film, a periodic change in refractive index is induced, and an incident probe beam is diffracted at $\sim 100 \%$ diffraction efficiency. The recorded holograms can be erased by thermal treatment or by photoirradiation with a single writing beam. Angular multiplicity is discussed from the viewpoint of high-density information storage.
\end{abstract}

Polymer Journal (2010) 42, 525-533; doi:10.1038/pj.2010.45; published online 26 May 2010

Keywords: azobenzene; grating; hologram; liquid crystal; refractive index

\section{INTRODUCTION}

Holograms, in which we can observe natural three-dimensional (3D) images without eyeglasses, have been of great interest from the viewpoint of 3D television. ${ }^{1-3}$ In addition, other photonic applications such as holographic memory, optical switch and waveguide have been proposed. $^{3-9}$ The holograms can be categorized into two types: (1) Raman-Nath type and (2) Bragg type. The Raman-Nath hologram recorded in a thin film causes multiple diffraction of an incident beam, leading to low diffraction efficiency $(<34 \%)$. On the other hand, the Bragg hologram shows a single diffraction, which enables $100 \%$ diffraction efficiency. Furthermore, angular multiplicity can be used in the Bragg hologram. Therefore, thick films are suitable for holograms with high diffraction efficiency. The diffraction efficiency also depends on a photoinduced change in refractive index of the film. If materials in which a large change in refractive index can be induced by light can be prepared, the performances parameters such as diffraction efficiency, response and multiplicity of the holograms can be enhanced. How can we induce the change in refractive index efficiently? In an isotropic material, the change in refractive index by photoirradiation is rather small and the efficiency is not high. In addition, the material is theoretically polarization inactive. On the other hand, an anisotropic material could show birefringence. By controlling their molecular alignment by photoirradiation, a large change in refractive index and high diffraction efficiency can be obtained. Furthermore, an anisotropic material shows polarization activity. Therefore, we can expect the fabrication of high-performance holograms by photoinduced alignment of anisotropic molecules.
Liquid crystals (LCs) show optical anisotropy and fluidity, and their molecular alignment is cooperatively varied by external stimuli. ${ }^{10}$ In other words, LCs can amplify the external stimuli by means of cooperative molecular motion. Therefore, LCs are widely used as LC displays, making use of their response to the electric field. Changes in birefringence are accompanied by the electric-field-induced changes in molecular alignment of the LCs. Transmittance of light can be reversibly controlled when the LCs are put between a pair of crossed polarizers. If LC molecules are driven by light, various photonic applications are realized in addition to the LC displays. However, most LC molecules show no significant response to the optical field.

An azobenzene, which is one of the common photochromic molecules, can be used as a photoresponsive trigger of the LCs. ${ }^{11}$ When the azobenzene is incorporated into the LC systems, the photoinduced change in molecular shape of the azobenzene causes the change in alignment of the surrounding LC molecules, which means that the change in molecular alignment is amplified by the cooperative motion. The azobenzene-containing LC systems have been developed to enable two-dimensional image storage and 3D image storage in Raman-Nath holograms in an early stage. ${ }^{4,12-30}$ Recently, holographic recording in thick polymer films has been explored, aimed at developing holographic memory with high-density information storage. ${ }^{31,32}$ In developing thick holograms known as Bragg holograms, angular multiplicity can be used. In addition, rewritable recording has been achieved, as the photoinduced changes are reversible using the azobenzene.

In this review, the concept of amplification of the photoinduced change in refractive index with azobenzene-containing LCs and their 
holographic applications are described. ${ }^{33-59}$ To enhance the photoinduced change in refractive index, highly birefringent LC polymers and LC block copolymers with nanostructures have been developed in the Raman-Nath holograms. ${ }^{4,17-28,60-70}$ In the Bragg holograms, fabrication of thick transparent polymer films is a key process. Several approaches to obtain high performance parameters of the Bragg holograms are introduced. ${ }^{8,9,31,32,71-75}$

\section{PHOTOINDUCED CHANGE IN MOLECULAR ALIGNMENT IN LCS}

To make LCs photoactive, azobenzene has been used. When a small amount of azobenzene is incorporated into the LCs, the trans form of the azobenzene, which has a rod-like shape, stabilizes the phase structure of the LC phase, ${ }^{33-35}$ while its isomer (cis form) is bent and tends to destabilize the phase structure of the mixture. As a result, the LC-isotropic phase transition temperature $\left(T_{\mathrm{c}}\right)$ of the mixture with the cis form $\left(T_{\mathrm{cc}}\right)$ is much lower than that with the trans form $\left(T_{\mathrm{ct}}\right)$. If the temperature of the sample $(T)$ is set at a temperature between $T_{\mathrm{ct}}$ and $T_{\mathrm{cc}}$, and the sample is irradiated to cause trans-cis photoisomerization of the azobenzene guest molecules, then $T_{\mathrm{c}}$ decreases with an accumulation of the cis form, and when $T_{\mathrm{c}}$ becomes lower than the irradiation temperature $T$, an LC-isotropic phase transition of the sample is induced (photochemical phase transition). Photochromic reactions are reversible, and with cis-trans back-isomerization of the azobenzene the sample reverts to the initial LC phase. This means that phase transitions of LC systems can be induced reversibly by photochemical reactions of photoresponsive guest molecules (Figure 1). Tazuke et al. ${ }^{34}$ reported an example of the photochemical nematic-isotropic phase transition in azobenzene/nematic LC mixtures. This concept is very simple, and can be applied to advanced systems based on polymers or other photochromic molecules. Control of the LC alignment with a photoactive surface layer, known as command surface, has been explored individually by Ichimura et al. ${ }^{36}$ and Gibbons et al. ${ }^{37}$ They used trans-cis photoisomerization of the azobenzene attached to the surface of the glass substrate to change the alignment of LCs.

The photochemical phase transition accompanies a large change in molecular alignment, which indicates that a huge change in refractive index is also induced depending upon the initial birefringence of the LCs. The relationship between the photochemical phase transition and refractive index of LCs was investigated by means of transmissionmode and reflection-mode analyses. It was revealed that the isotropicLC phase transition was accelerated in the reflection mode due to the fast recovery of the initial alignment near the surface. ${ }^{38-40}$ In addition, the value of the photoinduced change in refractive index was found to be $\sim 0.1$, which was much higher than those observed in other inorganic and organic materials.

A large change in molecular alignment can also be induced in LC polymers containing the azobenzene. Ikeda et al.,41-44 prepared various azobenzene-containing LC polymers and investigated their photoresponsive behavior (Figure 2).

In polymers, we can make use of the glass transition temperature $\left(T_{\mathrm{g}}\right)$, below which polymers are immobilized to hold the induced alignment for a long time. If the temperature is raised above $T_{\mathrm{g}}$, the molecular alignment induced by light is erased and returned to its initial state because of the molecular motion of the main chain in the polymer. Polymer systems require no glass cells, which are necessary to hold low-molecular-weight LCs. Polymer solutions can be spincoated onto a plate of glass substrate, which is much simpler compared with preparation of the low-molecular-weight LCs. When uniaxially aligned LC polymer films with the azobenzene are irradiated with ultraviolet

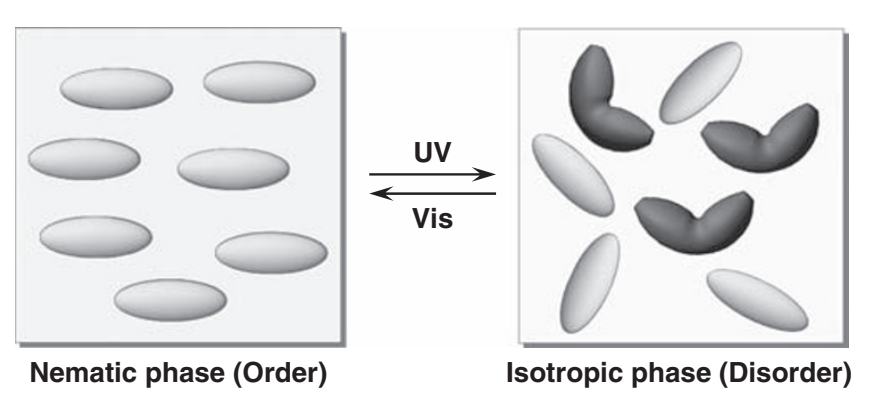

Figure 1 Photochemical phase transition of LCs doped with an azobenzene.
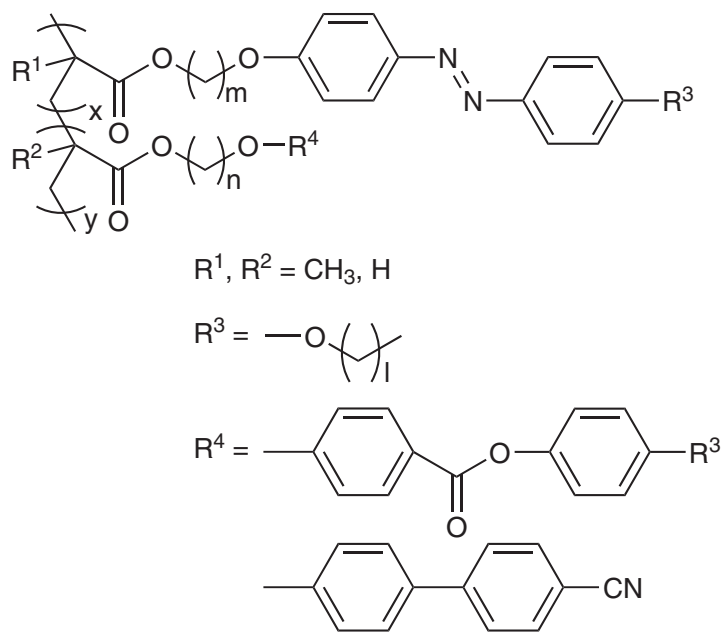

Figure 2 Chemical structures of azobenzene-containing LC polymers.

light through a photomask, LC-isotropic transition takes place only in the irradiated area. A bright image of the unirradiated area is observed, as only ordered molecules are optically anisotropic. The recorded two-dimensional images are temporally stable under the dark below $T_{\mathrm{g}}$. Photochemical reactions of the azobenzene occur on a timescale of picoseconds (ps). When the azobenzene LC polymer films were irradiated with a ps laser pulse, it was found that LC-isotropic phase transition takes place within $200 \mathrm{~ns}$ (Figure 3). ${ }^{4}$

Irradiation of the LC and amorphous polymer films with a linearly polarized beam also brings about a significant change in molecular alignment. Light absorption to give excited states of molecules complies with exclusive requirements, including the coincidence of the electric eld vector of light with the direction of a transition moment of the molecule (photoselection). For instance, trans-azobenzenes exhibit $\pi-\pi^{*}$ transition moments approximately parallel to the molecular long axis, whereby the trans-azobenzenes show angulardependent absorption of linearly polarized light: trans-azobenzene molecules with their transition moments parallel to the polarization direction of linearly polarized light are activated very effectively to their excited states, followed by trans-cis isomerization, whereas molecules with their transition moments perpendicular to the polarization direction of actinic light are inactive toward isomerization. After the repetition of trans-cis-trans isomerization cycles, once transazobenzene molecules have fallen perpendicular to the polarization direction of the actinic light, they become inactive toward the incident radiation. This means that at the end of the multicycles, there will be a net population of azobenzene molecules aligned perpendicular to the light polarization. This is well known as the 'Weigert effect'. ${ }^{45}$ Since the 


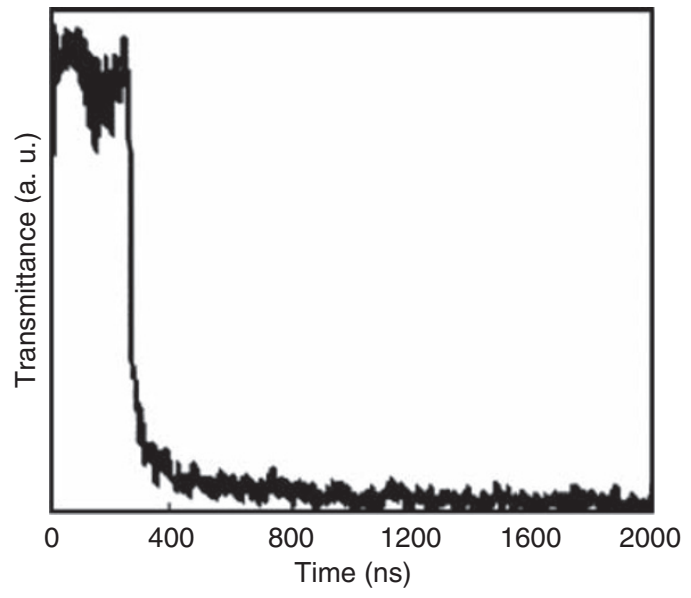

Figure 3 Time-resolved measurement of the photochemical phase transition of the azobenzene LC polymer.

first report by Weigert, there have been many studies published on this photoinduced anisotropy in the alignment of dye molecules. Among them, the work by Todorov et al., ${ }^{46}$ in which they showed a possibility of using a polymer containing azobenzene molecules as an optical recording medium, is a milestone in the research on dye-doped optical systems. Their system contained azo dyes, methyl red and methyl orange, dispersed in a poly(vinyl alcohol) matrix. When the dyedoped polymers are irradiated with a linearly polarized laser beam at $488 \mathrm{~nm}$, the optical transmittance for light polarized along the polarization direction of the actinic light increases, whereas that for light perpendicular to the direction of the actinic light decreases. Optical dichroism is created in this way owing to the anisotropic realignment of trans-azobenzene molecules. However, in this system the stability of the induced anisotropy was low: it remains unchanged only for a very short period even in the dark. This drawback was overcome by Natansohn et al., ${ }^{4-51}$ who developed amorphous azobenzene polymers, and by others. They succeeded in inducing stable birefringence in amorphous polymers at ambient temperatures below $T_{\mathrm{g}}$ by attaching the azobenzene moieties to polymers through covalent bonds. The induced alignment can be erased thermally and photochemically, and reinduced by irradiation with linearly polarized light. Photoinduction of molecular alignment has been extended to LC polymers possessing both mesogens and azobenzene moieties in the side chains. Several studies clearly showed that non-photoactive mesogens could undergo reorientation concomitantly with azobenzene moieties above $T_{\mathrm{g}}$, thanks to their cooperative motion. On the other hand, different arguments were presented regarding reorientation behavior below $T_{\mathrm{g}}{ }^{52,53}$ Work on the photoinduced alignment behavior of side-chain LC polymers has revealed that the dipoledipole interaction affecting cooperative motion is crucial in the reorientation process. ${ }^{54}$ The $3 \mathrm{D}$ manipulation of azobenzene moieties was explored by controlling the incident angle of the writing beam. ${ }^{55,56}$ Photocontrol of nanostructures in an LC block copolymer film was achieved using the Weigert effect of the azobenzene. ${ }^{57}$ Seki's group $^{58}$ prepared LC polymer brushes containing an azobenzene mesogen on a quartz or silicon substrate by surface-initiated atom transfer radical polymerization.

\section{RAMAN-NATH HOLOGRAMS OF LIQUID-CRYSTALLINE POLYMERS}

As mentioned above, LC polymers containing the azobenzene change their molecular alignment, and are suitable for holographic applica- tions, as a large change in refractive index can be expected. In holograms, photoinduced modulation of refractive index is formed in a material due to distribution of the light intensities created by interference of two laser beams. Wendorff et al. ${ }^{12,13}$ first used azobenzene LC polymers as a rewritable holographic media with a large change in refractive index, and successfully recorded 3D images. Ramanujam et al. ${ }^{18}$ recorded holographic gratings in thin azobenzene-containing peptide oligomer films by interference of orthogonal polarized beams, which form polarization gratings to obtain high diffraction efficiency. Modification of mesogenic moieties has been explored. To enhance the change in refractive index, highly birefringent LC polymers have been synthesized. Ikeda's group ${ }^{25,26}$ incorporated tolane moieties, which are known as a highly birefringent mesogen, and recorded 3D images (Figure 4). Furthermore, LC polymers with azotolane moieties in the side chain have been designed (Figure 5). ${ }^{27-30}$ In the azotolane core, $\pi$-electrons are delocalized through the phenyl rings, which leads to enhanced optical anisotropy.

When the uniaxially aligned polymer films were prepared on a glass substrate coated with a rubbed polyimide layer, significantly large birefringence was observed. ${ }^{27}$ The values of birefringence increased depending on the length of the mesogenic core, and the value of 0.76 was obtained for a five-ring azotolane LC polymer film. ${ }^{30}$ Polarized absorption spectra show a higher-order parameter of 0.71 in the azotolane moiety, leading to a very large birefringence (Figure 6).

Another interesting feature of the azotolane LC polymers is their high photosensitivity. When two laser beams were made to overlap in the three-ring azotolane LC polymer films, holographic gratings were formed even at a light intensity of several $\mathrm{mW} \mathrm{cm}^{-2} \cdot{ }^{27}$ Generally, LC polymers show less sensitivity compared with low-molecular-weight LCs, because the molecular motion of the polymer is suppressed. Holographic gratings are usually formed in the LC polymer films at the light intensity of several hundreds of $\mathrm{mW} \mathrm{cm}^{-2}$. The azotolane LC polymers possess both large birefringence and high sensitivity. Holographic formation in polymers with an azochromophore connected with a hetero ring has been explored from the viewpoint of a large change in refractive index.

Block copolymers have been used for holographic gratings in the Raman-Nath regime. ${ }^{32,59-62}$ Iyoda's group ${ }^{59}$ have reported that LC block copolymers composed of poly(ethylene oxide) and polymethacrylate blocks with the azobenzene moiety in the side chain show stable nanocylinder structures with no defects all over the film. In this system, the azobenzene moieties function as a mesogen to stabilize the nanocylinder structures. As the azobenzene is photoactive, photoinduced changes in alignment of the azobenzene mesogen take place, followed by realignment of the nanocylinder. Seki's group ${ }^{60}$ focused on the effect of film thickness on the direction of the nanocylinders, and applied it to the formation of the holographic gratings. It was also revealed that thermal treatment of the LC block copolymer films irradiated with interfering beams gave rise to significant enhancement of surface relief gratings. ${ }^{61}$ By overlapping the writing beams at a wide incident angle, fine periodic structures are formed in the azobenzene LC block copolymer film with a nanostructure. ${ }^{62}$ Nanogratings have been proposed as a new optical element. Ikeda's group ${ }^{57}$ explored the photocontrol of nanocylinder structures with a linearly polarized beam (Figure 7). The Weigert effect can be applied to the azobenzene LC block copolymer systems. Various other LC block copolymers have been developed, and wide ranging applications taking advantage of controlled nanostructures are expected. ${ }^{63-67}$

Nanoscale molecular alignment may have an important role in holographic performance. If molecules interact with each other on a nanoscale, the spatial distribution of the molecules affects holographic 


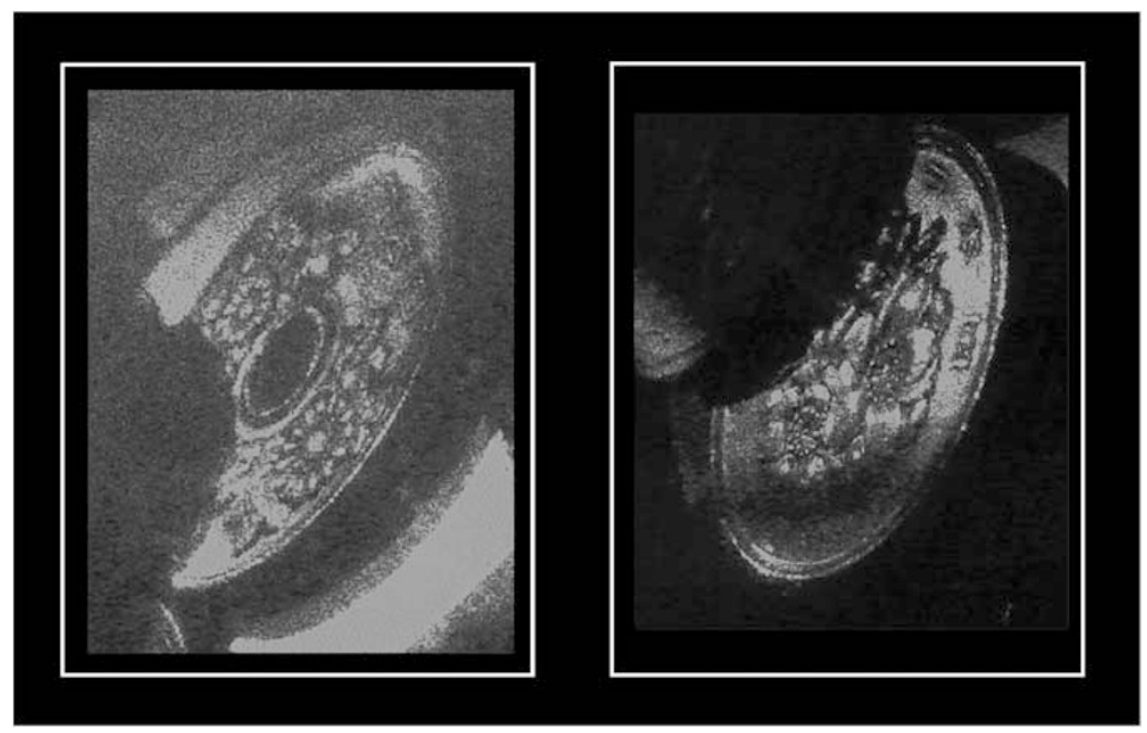

Figure 4 Three-dimensional reconstructed images in the azobenzene-containing LC polymers.
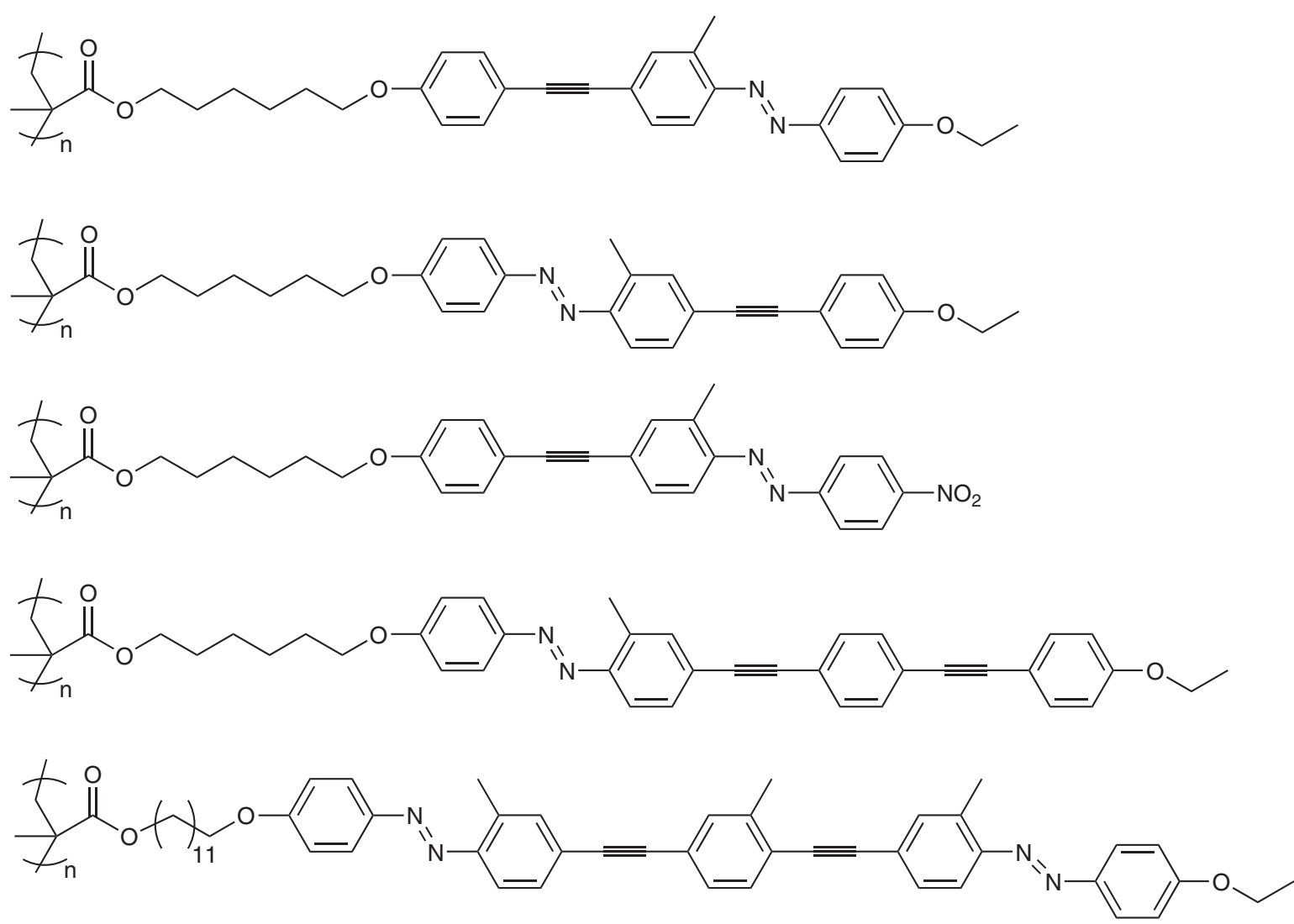

Figure 5 Chemical structures of azotolane LC polymers with high birefringence.

formation. For example, we can expect that a block copolymer tends to show an LC phase and induces a larger change in birefringence compared with a random copolymer with a similar composition ratio. Block and random copolymers with similar contents of the azobenzene mesogen were prepared, and their thermodynamic properties and photoresponsive behavior were investigated. ${ }^{68}$ Even with similar content the block copolymer showed a distinct LC phase, while the random copolymer was amorphous, which clearly indicates the effect of the nanoscale spatial molecular arrangement. When holograms were recorded in thin block and random copolymer films, the photoinduced change in refractive index was greatly enhanced only in the block copolymer film, owing to its LC nature.

Optical devices for optical communications have been proposed by means of the Raman-Nath hologram. ${ }^{69,70}$ Grating waveguide couplers 
with a flat surface were fabricated in an azobenzene LC polymer film by holographic lithography using $\mathrm{Ar}^{+}$laser beams at $488 \mathrm{~nm}$. When a probe beam at $633 \mathrm{~nm}$ was incident on one grating of a grating waveguide coupler, the beam propagated in the waveguide and an output beam emerged from the other grating with a throughput coupling efficiency of 5\%. ${ }^{69}$ Upon irradiation of the film between two gratings with ultraviolet light to cause trans-cis photoisomerization of the azobenzene moiety, the intensity of the output beam was repeatedly switched. It was found that the alternating irradiation at 366 and $436 \mathrm{~nm}$ induced reversible changes in the intensity of the guided probe beam.

\section{BRAGG HOLOGRAMS OF LIQUID-CRYSTALLINE POLYMERS}

As mentioned above, diffraction efficiency depends on film thickness and change in refractive index. If thick films can be obtained with a material capable of inducing a large change in refractive index, high diffraction efficiency can be expected. However, it is not easy to fabricate a high-performance Bragg hologram with the conventional azobenzene-containing LC polymers because of extensive light scattering owing to their LC nature. In addition, absorbance is usually so high that incident writing beams cannot penetrate deep into the thick films. The concentration of the azobenzene moiety must be suppressed, which lowers the change in refractive index. There is a tradeoff problem between the content of the azobenzene and change in refractive index. Larger changes in refractive index need to be induced even at a lower content of the azobenzene. To solve these problems, Schmidt's group ${ }^{32,71,72}$ designed polymer blends of block copolymers

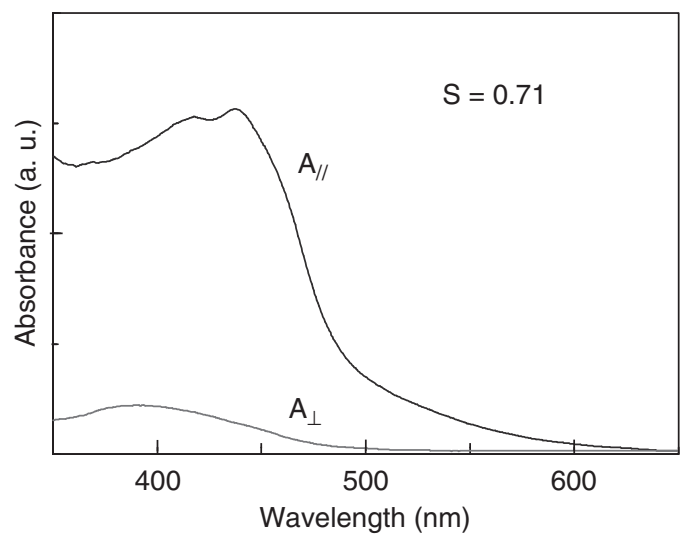

Figure 6 Polarized absorption spectra and estimated order parameter $(S)$ of uniaxially aligned azotolane LC polymers. and polystyrene to obtain thick films and generate Bragg holograms with high angular multiplicity. Minabe et al. ${ }^{31}$ used blends of an azobenzene-containing polyester and a polyester with a photoinactive moiety to prepare transparent thick films.

Amorphous random copolymers with functional components have also been designed. ${ }^{73-75}$ The holographic performance of the systems can be optimized just by a change in the combination of components. The systems consist of three components: (1) a photoresponsive component, (2) an amplifying component and (3) a transparent component. Examples of the chemical structures and properties of the polymers are shown in Figure 8. The azobenzene functions as the photoresponsive component. The anisotropic moiety functions as the amplifying component to amplify the change in refractive index triggered by the azobenzene. Finally, the transparent component prevents the light scattering and controls $T_{\mathrm{g}}$. Each component can be designed individually to maximize the change in refractive index. In the photoresponsive component, the terminal groups at the azobenzene have an important role in photoisomerization and the resultant alignment change. In the amplifying component, an increase in the molecular refraction of the anisotropic moiety is effective in amplifying the photoinduced change in the photoresponsive unit. Transparent thick polymer films were successfully fabricated by optimizing the combination of components and their composition ratio. A schematic illustration of the preparation of the films is shown in Figure 8c.

In the conventional LC polymer films, a large change in refractive index is induced; however, the light scattering is a serious problem. The polymers with functional components show no LC phase macroscopically, in which the light scattering is eliminated. In addition, microscopic interaction between photoresponsive and amplifying components can be expected to enhance the change in refractive index.

Holograms were generated by overlapping two writing beams from an $\mathrm{Ar}^{+}$laser in the film, and the light intensities of transmitted and diffracted probe beams from a He-Ne laser were measured with photodetectors. Here, the diffraction efficiency was defined as the ratio of the light intensity of the diffracted beam to that of the incident beam.

Figure 9 shows an example of the holographic recording. Upon irradiation with the writing beams, diffraction efficiency increased to $\sim 100 \%$, which is the theoretical maximum, and in contrast, the transmittance decreased, which means that the incident probe beam is diffracted with no scattering loss.

The effect of the structure of the azobenzene moieties has been studied (Figure 10). Azobenzene moieties with a different terminal group, such as ethoxy, cyano and nitro groups, were used, keeping the

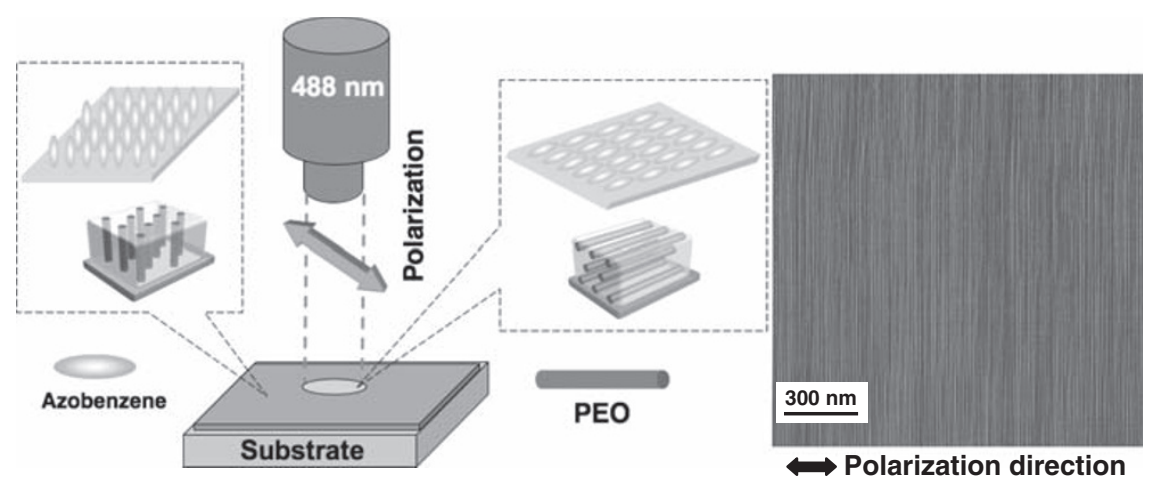

Figure 7 Photocontrol of nanostructures in the azobenzene-containing LC block copolymer. 


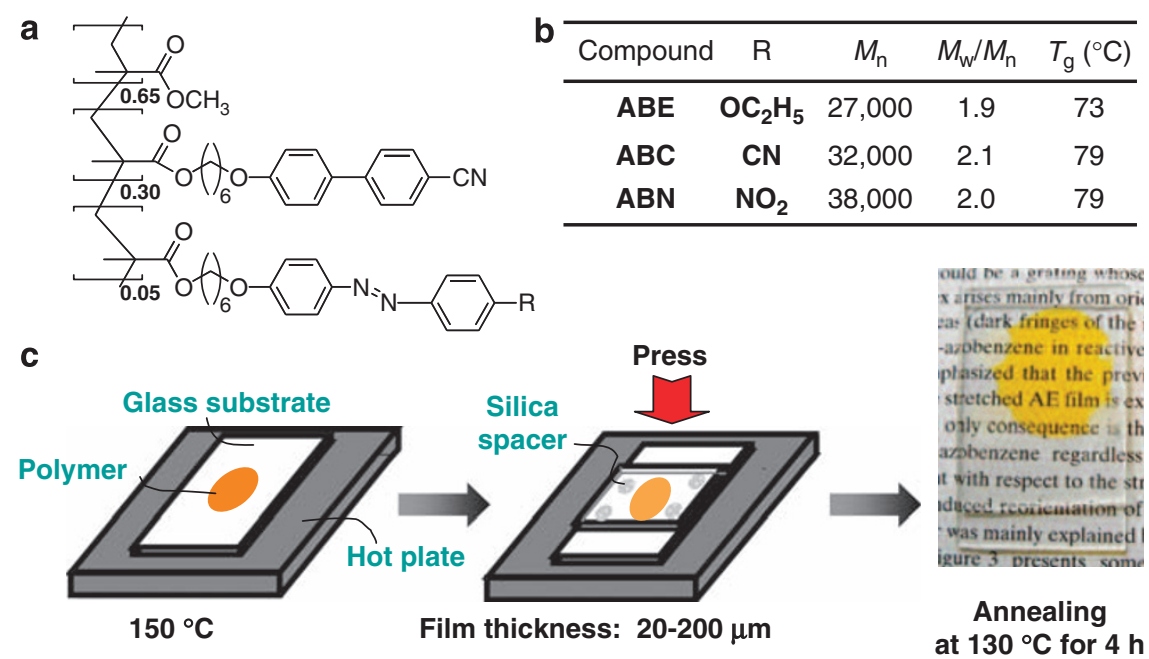

Figure 8 Chemical structures (a) and properties (b) of azobenzene-containing copolymers with functional components, and preparation of thick transparent film (c).

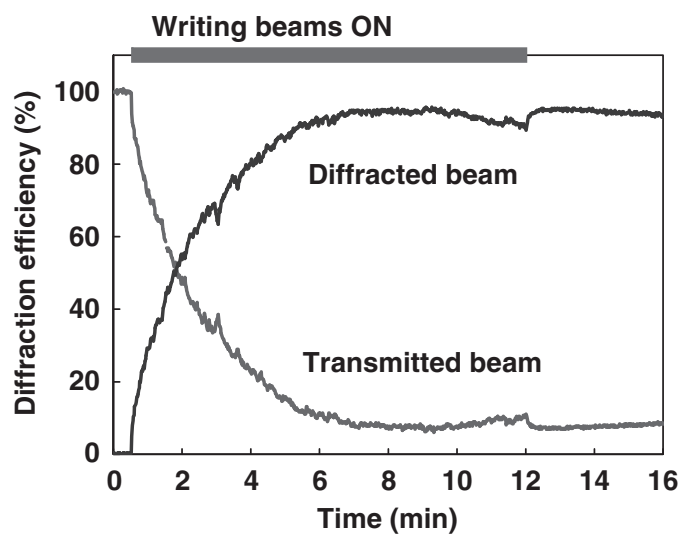

Figure 9 Change in diffraction efficiency as a function of time.

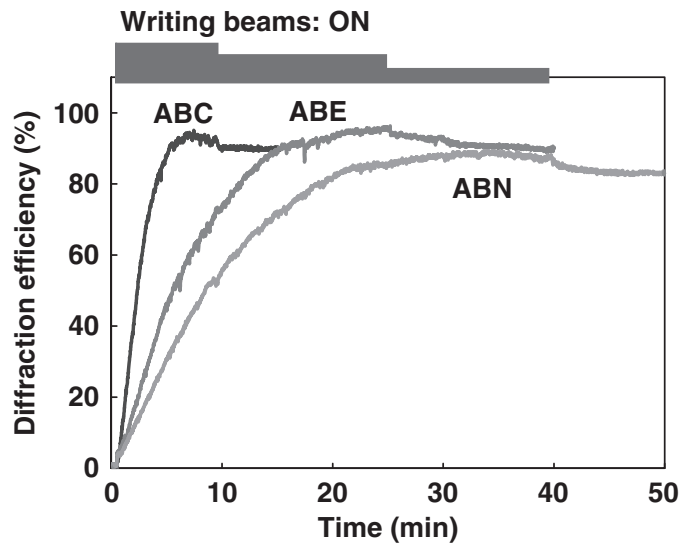

Figure 10 Effect of terminal groups of the azobenzene on diffraction efficiency.

composition ratio of the components the same. The fastest response was obtained in the azobenzene with the cyano group.

The effect of the amplifying component was also investigated. ${ }^{74}$ Four types of polymers with the azobenzene at the same content and

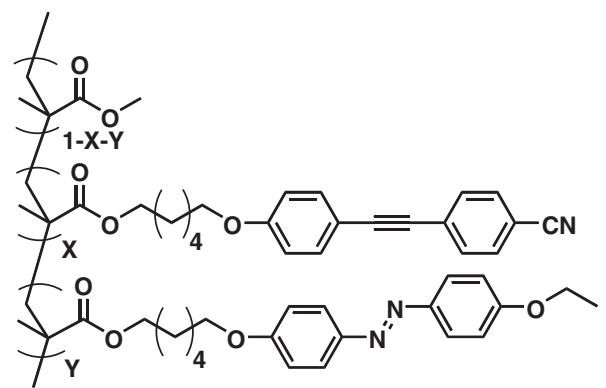

\begin{tabular}{lccccc}
\hline Compound & $\mathrm{X}$ & $\mathrm{Y}$ & $M_{\mathrm{n}}$ & $M_{\mathrm{w}} / M_{\mathrm{n}}$ & $T_{\mathrm{g}}\left({ }^{\circ} \mathrm{C}\right)$ \\
\hline MT0A5 & 0 & 0.05 & 25,000 & 3.9 & 99 \\
MT20A5 & 0.20 & 0.05 & 22,000 & 2.0 & 74 \\
MT30A5 & 0.30 & 0.05 & 32,000 & 2.2 & 77 \\
MT40A5 & 0.40 & 0.05 & 30,000 & 1.9 & 61 \\
\hline
\end{tabular}

Figure 11 Chemical structures and properties of polymers with a tolane moiety as the amplifying component.

the tolane moiety at contents of $0,20,30$ and $40 \%$ were prepared (Figure 11). The tolane moiety is known as a highly birefringent mesogenic core and could function as the amplifying component. The content of the azobenzene was maintained as $5 \%$ to exclude the effect of the photoresponsive component.

As shown in Figure 12, the diffraction efficiency increased with an increase in content of the tolane moiety. This result clearly indicates that the tolane moiety amplifies the change in refractive index even if those polymers show no LC phase macroscopically. Nanoscale interaction between the photoresponsive and amplifying components is implied. A schematic illustration of molecular arrangements in the hologram is shown in Figure 13. In the initial state, the molecular alignment is random. Upon irradiation with interfering beams, the azobenzene moieties become aligned in the bright area of the interference pattern, followed by alignment of the amplifying component. The recorded hologram was observed by polarizing optical microscopy to confirm the mechanism. In fact, the film showed bright fringes at 
every $45^{\circ}$ when rotated, which means that anisotropic molecular alignment is induced in the holograms. ${ }^{74}$

Angular multiplicity, which is characteristic of the Bragg hologram, is important for high-density data storage. To record multiple holograms in a single spot, the first hologram is recorded and then the film is rotated to record a next hologram in the same spot. By repeating this procedure, Ikeda's group ${ }^{73}$ recorded 55 holograms in a single spot. Schmidt's group ${ }^{71}$ achieved 80 holograms with polymer blends. Optimization of the systems might help make further advances in angular multiplicity. Recently, a combination of the angular multiplicity with the polarization direction has been explored. After the first hologram is recorded with s-polarized beams, the other hologram can be recorded with p-polarized beams in the same spot as the same incident angle of the writing beams. The hologram recorded with s-polarized writing beams is read out only with an s-polarized probe beam, while it is not detected with an p-polarized probe beam (Figure 14). Therefore, holograms can be recorded and probed at various angles with two polarized beams in a single spot. Ten

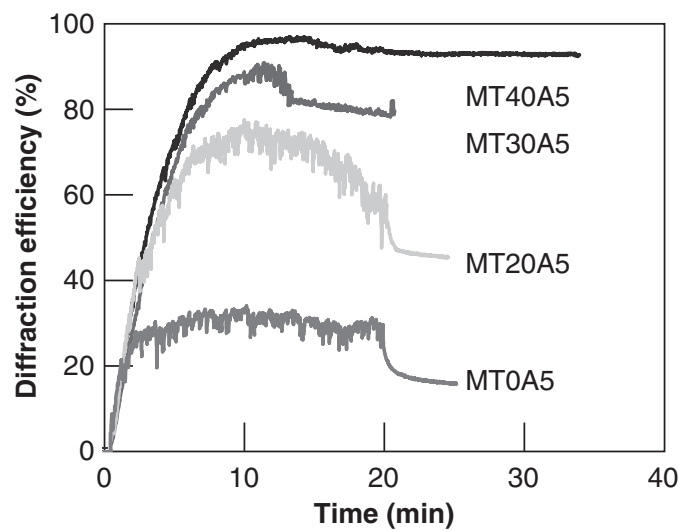

Figure 12 Effect of the tolane content on diffraction efficiency. holograms have been reported so far in rewritable polymer systems. Such polarization activity can be realized by means of anisotropic photochromic materials.

The polarization state of the writing beams can also be used. $^{14,15,74,76}$ If two laser beams interfere, various interference patterns are observed depending on the polarization state. For example, parallel linearly polarized beams, such as a combination of s,s-polarization, give intensity gratings. The light intensity changes periodically in the intensity gratings. On the other hand, if orthogonal polarized beams interfere, polarization gratings, in which only the polarization state changes periodically keeping light intensity uniform, are formed. The recorded hologram also depends on the interference patterns. In the hologram recorded with orthogonal polarized beams, a totally different molecular arrangement is obtained. Uniform fringes are observed at any rotation angle, which can be explained by the in-plane circular arrangements of the photoresponsive and amplifying

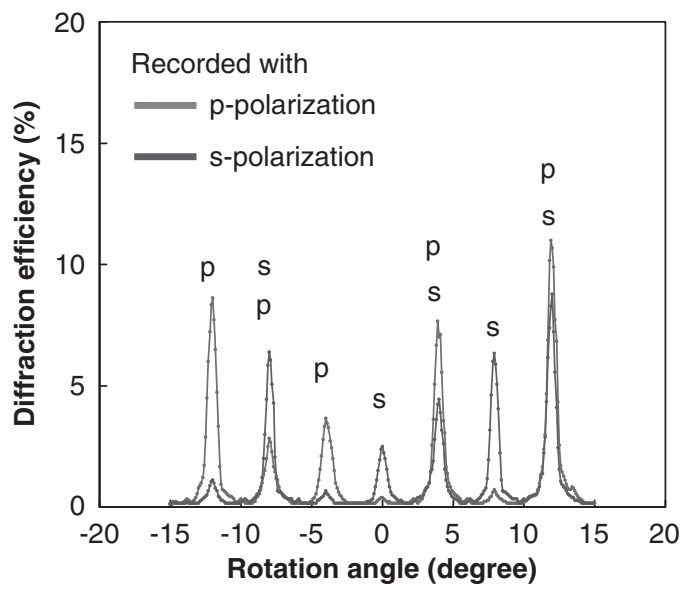

Figure 14 Combination of angular multiplicity and polarization multiplicity in the Bragg hologram.

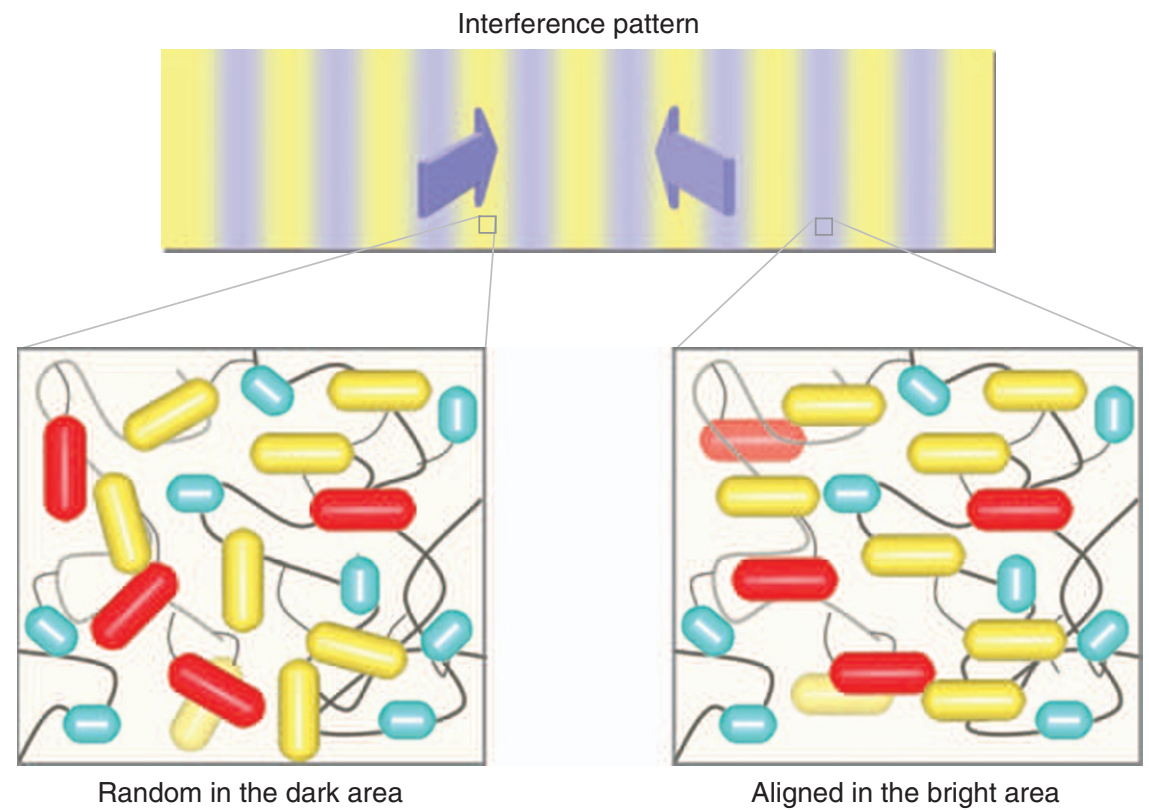

Figure 13 Schematic illustration of the molecular arrangement in the hologram. 
molecules. It is noteworthy that the polarization Bragg hologram acts as a polarized beam splitter. If a linearly polarized beam is incident on the film, right- and left-circular polarized beams can be separated at $\sim 100 \%$ efficiency. In the polarization holograms, the performance parameters are enhanced by the efficient photoinduced change in molecular alignment. The value of the photoinduced change in refractive index reaches $10^{-2} \cdot{ }^{74}$ Furthermore, 90\% diffraction efficiency was achieved within $900 \mathrm{~ms}$ at a light intensity of $2000 \mathrm{~mW} \mathrm{~cm}^{-2}$. The recorded holograms were erased by thermal treatment or photoirradiation with a single writing beam.

\section{CONCLUSION}

For more than 20 years, LC polymers containing the azobenzene moiety have been extensively developed, aiming at photonic applications such as optical communication, holographic memory, 3D television and so on. Among them, the hologram has still been one of the attractive applications. Recently, holographic performance has also been improved with regard to diffraction efficiency, response time and multiplicity by development of newly designed polymer systems. Particularly, the photoinduced changes in refractive index have increased remarkably. Concurrent studies in development of new recording methods and holographic devices will stimulate further progress of LC polymer materials.

\section{ACKNOWLEDGEMENTS}

I thank the following colleagues for their guidance and experimental contribution: Professors Tomiki Ikeda, Takeshi Shiono, Akihiko Kanazawa, Osamu Tsutsumi, Tomokazu Iyoda, Drs Haifeng Yu, Hideo Kurihara, Kunihiko Okano, Chong-Uk Bang, Mrs Daisuke Sato, Hyo-Bok Cha, Makoto Ishiguro, and Mses Aya Saishoji and Yumiko Naka.

1 Gabor, D. A new microscopic principles. Nature 161, 777-778 (1948).

2 Kogelnik, H. Coupled wave theory for thick hologram gratings. Bell Syst. Technol. J. 48, 2909-2946 (1969).

3 Bieringer, T. in Holographic Data Storage (eds. Coufal, H. J., Psaltis, D., \& Sincerbox, G. T.) Springer Series in Optical Sciences, Springer 209 (New York, 2000).

4 Ikeda, T. Photomodulation of liquid crystal orientations for photonic applications. J. Mater. Chem. 13, 2037-2057 (2003).

5 Nathanson, A. \& Rochon, P. Photoinduced motions in azo-containing polymers. Chem. Rev. 102, 4139-4176 (2002).

6 Hagen, R. \& Bieringer, T. Photoaddressable polymers for optical data storage. Adv. Mater. 13, 1805-1810 (2001).

7 Ichimura, K. Photoalignment of liquid-crystal systems. Chem. Rev. 100, 1847-1874 (2000).

8 Matharu, A. S., Jeeva, S. \& Ramanujam, P. S. Liquid crystals for holographic optical data storage. Chem. Soc. Rev. 36, 1868-1880 (2007).

9 Hvilsted, S., Sánchez, C. \& Alcalá, R. The volume holographic optical storage potential in azobenzene containing polymers. J. Mater. Chem. 19, 6641-6648 (2009).

10 Gray, G. W. in Handbook of Liquid Crystals, Vol 1, Fundamentals (eds. Demus, D., Goodby, J., \& Gray, G. W., Spiess, H. W., \& Vill, V.) Wiley-VCH (Weinheim, 1998).

11 Irie, M. Photoresponsive polymers. Adv. Polym. Sci. 94, 27-67 (1990).

12 Eich, M. \& Wendorff, J. H. Reversible digital and holographic optical storage in polymeric liquid-crytals. Makromol. Chem., Rapid Commun. 8, 59-63 (1987).

13 Eich, M. \& Wendorff, J. H. Erasable holograms in polymeric liquid-crytals. Makromol. Chem., Rapid Commun. 8, 467-471 (1987).

14 Todorov, T., Nikolova, L. \& Tomova, N. Polarization holography. 1: a new highefficiency organic material with reversible photoinduced birefringence. Appl. Opt. 23, 4309-4312 (1984).

15 Todorov, T., Nikolova, L. \& Tomova, N. Polarization holography. 2: polarization holographic gratings in photoanisotropic materials with and without intrinsic birefringence. Appl. Opt. 23, 4588-4591 (1984).

16 Todorov, T., Nikolova, L., Stoyanova, K. \& Tomova, N. Polarization holography. 3: some applications of polarization holographic recording. Appl. Opt. 24, 785-788 (1985).

17 Hvilsted, S., Andruzzi, F., Kulinna, C., Siesler, H. W. \& Ramanujam, P. S. Novel sidechain liquid crystalline polyester architecture for reversible optical storage. Macromolecules 28, 2172-2183 (1995).

18 Berg, R. H., Hvilsted, S. \& Ramanujam, P. S. Peptide oligomers for holographic data storage. Nature 383, 505-508 (1996).
19 Ramanujam, P. S., Holme, N. C. R. \& Hvilsted, S. Atomic force and optical near-field microscopic investigations of polarization holographic gratings in a liquid crystalline azobenzene side-chain polyester. Appl. Phys. Lett. 68, 1329-1331 (1996).

20 Rasmussen, P. H., Ramanujam, P. S., Hvilsted, S. \& Berg, R. H. A remarkably efficient azobenzene peptide for holographic information storage. J. Am. Chem. Soc. 121, 4738-4743 (1999).

21 Hasegawa, M., Yamamoto, T., Kanazawa, A., Shiono, T. \& Ikeda, T. A dynamic grating using a photochemical phase transition of polymer liquid crystals containing azobenzene derivatives. Adv. Mater. 11, 675-677 (1999).

22 Hasegawa, M., Yamamoto, T., Kanazawa, A., Shiono, T. \& Ikeda, T. Photochemically induced dynamic grating by means of side chain polymer liquid crystals. Chem. Mater. 11, 2764-2769 (1999).

23 Yamamoto, T., Hasegawa, M., Kanazawa, A., Shiono, T. \& Ikeda, T. Phase-type gratings formed by photochemical phase transition of polymer azobenzene liquid crystals: enhancement of diffraction efficiency by spatial modulation of molecular alignment. J. Phys. Chem. B 103, 9873-9878 (1999).

24 Yamamoto, T., Hasegawa, M., Kanazawa, A., Shiono, T. \& Ikeda, T. Holographic gratings and holographic image storage via photochemical phase transitions of polymer azobenzene liquid-crystal films. J. Mater. Chem. 10, 337-342 (2000).

25 Yoneyama, Y., Yamamoto, T., Hasegawa, M., Tsutsumi, O., Kanazawa, A., Shiono, T. \& Ikeda, T. Formation of intensity grating in a polymer liquid crystal with a side-chain azobenzene moiety by photoinduced alignment change of mesogens. J. Mater. Chem. 11, 3008-3013 (2001).

26 Yoneyama, Y., Yamamoto, T., Hasegawa, M., Tsutsumi, O., Kanazawa, A., Shiono, T. \& Ikeda, T. High-performance material for holographic gratings by means of a photoresponsive polymer liquid crystal containing a tolane moiety with high birefringence. Macromolecules 35, 8751-8758 (2002).

27 Okano, K., Shishido, A., Tsutsumi, O., Shiono, T. \& Ikeda, T. Highly birefringent liquidcrystalline polymers for photonic applications: synthesis of liquid-crystalline polymers with side-chain azo-tolane mesogens and their holographic properties. J. Mater. Chem. 15, 3395-3401 (2005).

28 Okano, K., Shishido, A. \& Ikeda, T. Photochemical phase transition behavior of highly birefringent azotolane liquid-crystalline polymer films: effects of the position of the tolane group and the donoracceptor substituent in the mesogen. Macromolecules 39, 145-152 (2006).

29 Okano, K., Shishido, A. \& Ikeda, T. An azotolane liquid-crystalline polymer exhibiting extremely large birefringence and its photoresponsive behavior. Adv. Mater. 18, 523-527 (2006).

30 Okano, K., Tsutsumi, O., Shishido, A. \& Ikeda, T. Azotolane liquid-crystalline polymers: huge change in birefringence by photoinduced alignment change. J. Am. Chem. Soc. 128, 15368-15369 (2006).

31 Minabe, J., Maruyama, T., Yasuda, S., Kawano, K., Hayashi, K. \& Ogasawara, Y. Design of dye concentrations in azobenzene-containing polymer films for volume holographic storage. Jpn. J. Appl. Phys. 43, 4964-4967 (2004).

32 Häckel, M., Kador, L., Kropp, D., Frenz, C. \& Schmidt, H. W. Holographic gratings in diblock copolymers with azobenzene and mesogenic side groups in the photoaddressable dispersed phase. Adv. Funct. Mater. 15, 1722-1727 (2005).

33 Sackmann, E. Photochemically induced reversible color changes in cholesteric liquid crystals. J. Am. Chem. Soc. 93, 7088-7090 (1971).

34 Tazuke, S., Kurihara, S. \& Ikeda, T. Amplified image recording in liquid crystal media by means of photochemically triggered phase transition. Chem. Lett. 16, 911-914 (1987).

35 Ikeda, T., Sasaki, T. \& Ichimura, K. Photochemical switching of polarization in ferroelectric liquid-crystal films. Nature 361, 428-430 (1993).

36 Ichimura, K., Suzuki, Y., Seki, T., Kawanishi, Y. \& Aoki, K. Reversible change in alignment mode of nematic liquid crystals regulated photochemically by command surfaces modified with an azobenzene monolayer. Langmuir 4, 1214-1216 (1988).

37 Gibbons, W. M., Shannon, P. J., Sun, S. T. \& Swetlin, B. J. Surface-mediated alignment of nematic liquid crystals with polarized laser light. Nature 351, 49-50 (1991).

38 Shishido, A., Tsutsumi, O., Kanazawa, A., Shiono, T., Ikeda, T. \& Tamai, N. Rapid optical switching by means of photoinduced change in refractive index of azobenzene liquid crystals detected by reflection-mode analysis. J. Am. Chem. Soc. 119, 7791-7796 (1997).

39 Shishido, A., Tsutsumi, O., Kanazawa, A., Shiono, T., Ikeda, T. \& Tamai, N. Distinct photochemical phase transition behavior of azobenzene liquid crystals evaluated by reflection-mode analysis. J. Phys. Chem. B 101, 2806-2810 (1997).

40 Shishido, A., Kanazawa, A., Shiono, T., Ikeda, T. \& Tamai, N. Enhancement of stability in optical switching of photosensitive liquid crystal by means of reflection mode analysis. J. Mater. Chem. 9, 2211-2213 (1999).

41 Ikeda, T., Horiuchi, S., Karanjit, D. B., Kurihara, S. \& Tazuke, S. Photochemically induced isothermal phase transition in polymer liquid crystals with mesogenic phenyl benzoate side chains. 1. Calorimetric studies and order parameters. Macromolecules 23, 36-42 (1990).

42 Ikeda, T., Horiuchi, S., Karanjit, D. B., Kurihara, S. \& Tazuke, S. Photochemically induced isothermal phase transition in polymer liquid crystals with mesogenic phenyl benzoate side chains. 2 . Photochemically induced isothermal phase transition behaviors. Macromolecules 23, 42-48 (1990).

43 Ikeda, T., Karanjit, D. B., Kurihara, S. \& Tazuke, S. Photochemically induced isothermal phase transition in polymer liquid crystals with mesogenic cyanobiphenyl side chains. Macromolecules 23, 3938-3943 (1990).

44 Ikeda, T. \& Tsutsumi, O. Optical switching and image storage by means of azobenzene liquid-crystal films. Science 268, 1873-1875 (1995). 
45 Weigert, F. Uber Einen Neuen Effekt der Strahlung in Lichtempfindlichen Schichten. Verh. Dtsch. Phys. Ges. 21, 479-491 (1919).

46 Todorov, T., Tomova, N. \& Nikolova, L. High-sensitivity material with reversible photoinduced anisotropy. Opt. Commun. 47, 123-126 (1983).

47 Rochon, P., Gosselin, J., Natansohn, A. \& Xie, S. Optically induced and erased birefringence and dichroism in azoaromatic polymers. Appl. Phys. Lett. 60, 4-5 (1992).

48 Ho, M. S., Natansohn, A. \& Rochon, P. Azo polymers for reversible optical storage. 7. The effect of the size of the photochromic groups. Macromolecules 28, 6124-6127 (1995).

49 Song, O. K., Wang, C. H. \& Pauley, M. A. Dynamic processes of optically induced birefringence of azo compounds in amorphous polymers below Tg. Macromolecules 30, 6913-6919 (1997).

50 Buffeteau, T., Natansohn, A., Rochon, P. \& Pézolet, M. Study of cooperative side group motions in amorphous polymers by time dependent infrared spectroscopy. Macromolecules 29, 8783-8790 (1996).

51 Natansohn, A., Rochon, P., Meng, X., Barrett, C., Buffeteau, T., Bonenfant, S. \& Pézolet, M. Molecular addressing? Selective photoinduced cooperative motion of polar ester groups in copolymers containing azobenzene groups. Macromolecules $\mathbf{3 1}$, 1155-1161 (1998).

52 Anderle, K., Birenheide, R., Werner, M. J. A. \& Wendorff, J. H. Molecular addressing? Studies on light-induced reorientation in liquid-cystalline side-chain polymers. Liq. Cryst. 9, 691-699 (1991).

53 Wiesner, U., Reynolds, N., Roeffel, C. \& Spiess, H. W. Photoinduced reorientation in liquid-crystalline polymers below the glass transition temperature studied by time-dependent infrared spectroscopy. Makromol. Chem. Rapid Commun. 12, 457-464 (1991).

54 Stumpe, J., Läsker, L., Fischer, Th., Rutloh, M., Kostromin, S. \& Ruhmann, R. Photoorientation in amorphous and aligned films of photochromic liquid crystalline polymers. Thin Solid Films 284, 252-256 (1996).

55 Ichimura, K., Morino, S. \& Akiyama, H. Three-dimensional orientational control of molecules by slantwise photoirradiation. Appl. Phys. Lett. 73, 921-923 (1999).

$56 \mathrm{Wu}$, Y., Ikeda, T. \& Zhang, Q. Three-dimensional manipulation of an azo polymer liquid crystal with unpolarized light. Adv. Mater. 11, 300-302 (1999).

$57 \mathrm{Yu}, \mathrm{H}$., lyoda, T. \& Ikeda, T. Photoinduced alignment of nanocylinders by supramolecular cooperative motions. J. Am. Chem. Soc. 128, 11010-11011 (2006).

58 Uekusa, T., Nagano, S. \& Seki, T. Highly ordered in-plane photoalignment attained by the brush architecture of liquid crystalline azobenzene polymer. Macromolecules 42, 312-318 (2009).

59 Tian, Y., Watanabe, K., Kong, X., Abe, J. \& Iyoda, T. Synthesis, nanostructures, and functionality of amphiphilic liquid crystalline block copolymers with azobenzene moieties. Macromolecules 35, 3739-3747 (2002).

60 Morikawa, Y., Nagano, S., Watanabe, K., Kamata, K., Iyoda, T. \& Seki, T. Optical alignment and patterning of nanoscale microdomains in a block copolymer thin film. Adv. Mater. 18, 883-886 (2006).

61 Yu, H. F., Okano, K., Shishido, A., Ikeda, T., Kamata, K., Komura, M. \& Iyoda, T. Enhancement of surface-relief gratings recorded on amphiphilic liquid-crystalline diblock copolymer by nanoscale phase separation. Adv. Mater. 17, 2184-2188 (2005).

62 Yu, H., Shishido, A. \& Ikeda, T. Subwavelength modulation of surface relief and refractive index in preirradiated liquid-crystalline polymer films. Appl. Phys. Lett. 92, 103117 (2008).

$63 \mathrm{Yu}, \mathrm{H} ., \mathrm{Li}$, J., Ikeda, T. \& Iyoda, T. Macroscopic parallel nanocylinder array fabrication using a simple rubbing technique. Adv. Mater. 18, 2213-2215 (2006).

64 Yu, H., Shishido, A., Ikeda, T. \& Iyoda, T. Novel amphiphilic diblock and triblock liquidcrystalline copolymers with well-defined structures prepared by atom transfer radical polymerization. Macromol. Rapid Commun. 26, 1594-1598 (2005).

$65 \mathrm{Yu}$, H., Shishido, A., Iyoda, T. \& Ikeda, T. Novel wormlike nanostructures selfassembled in a well defined liquid crystalline diblock copolymer with azobenzene moieties. Macromol. Rapid Commun. 28, 927-931 (2007).

66 Yu, H., Shishido, A., Li, J., Kamata, K., Iyoda, T. \& Ikeda, T. Stable macroscopic nanocylinder arrays in an amphiphilic diblock liquid-crystalline copolymer with successive hydrogen bonds. J. Mater. Chem. 17, 3485-3488 (2007).

$67 \mathrm{Yu}, \mathrm{H}$., Asaoka, S., Shishido, A., lyoda, T. \& Ikeda, T. Photoinduced nanoscale cooperative motion in a well-defined triblock copolymer. Small 3, 768-771 (2007).

68 Yu, H., Naka, Y., Shishido, A. \& Ikeda, T. Well-defined liquid-crystalline diblock copolymers with an azobenzene moiety: synthesis, photoinduced alignment and their holographic properties. Macromolecules 41, 7959-7966 (2008).

69 Bang, C. U., Shishido, A. \& Ikeda, T. Azobenzene liquid-crystalline polymer for optical switching of grating waveguide couplers with a flat surface. Macromol. Rapid Commun. 28, 1040-1044 (2007).

70 Kurihara, H., Shishido, A. \& Ikeda, T. Evaluation of photoinduced change in refractive index of a polymer film doped with an azobenzene liquid crystal by means of a prismcoupling method. J. Appl. Phys. 98, 083510 (2005).

71 Häckel, M., Kador, L., Kropp, D. \& Schmidt, H. W. Polymer blends with azobenzenecontaining block copolymers as stable rewritable volume holographic media. Adv. Mater. 19, 227-231 (2007).

72 Breiner, T., Kreger, K., Hagen, R., Häckel, M., Kador, L., Müller, A. H. E., Kramer, E. J. \& Schmidt, H.- W. Blends of poly(methacrylate) block copolymers with photoaddressable segments. Macromolecules 40, 2100-2108 (2007).

73 Saishoji, A., Sato, D., Shishido, A. \& Ikeda, T. Formation of bragg gratings with large angular multiplicity by means of the photoinduced reorientation of azobenzene copolymers. Langmuir 23, 320-326 (2007).

74 Ishiguro, M., Sato, D., Shishido, A. \& Ikeda, T. Bragg-type polarization gratings formed in thick polymer films containing azobenzene and tolane moieties. Langmuir 23, 332-338 (2007).

75 Shishido, A., Ishiguro, M. \& Ikeda, T. Circular arrangement of mesogens induced in bragg-type polarization holograms of thick azobenzene copolymer films with a tolane moiety. Chem. Lett. 36, 1146-1147 (2007).

76 Ono, H., Sekiguchi, T., Emoto, A., Shioda, T. \& Kawatsuki, N. Light wave propagation and Bragg diffraction in thick polarization gratings. Jpn. J. Appl. Phys. 47, 7963-7967 (2008).

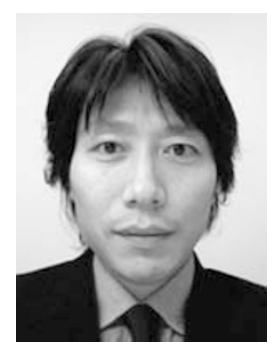

Atsushi Shishido received BS (1994), MS (1996) and PhD (1999) degrees from the Tokyo Institute of Technology under the guidance of Prof Tomiki Ikeda. In 2001, he became Assistant Professor at the Tokyo Institute of Technology after he spent 2 years as a postdoctoral research fellow, working with Profs IC Khoo and TE Mallouk in the Pennsylvania State University. He was promoted as Lecturer (2003) and as Associate Professor (2008) at the Tokyo Institute of Technology. He received the Award for Encouragement of Research in Polymer Science by the Society of Polymer Science, Japan (2005) and SPSJ Hitachi Chemical Award (2009). His research interests include design and application of photofunctional polymer materials. 